\title{
Physiological Emergence of Amyloid- $\beta$ During the Acute Cerebral Stress of Mechanical Ventilation Reduces Peri-Hippocampal and Cortical Blood-Brain Barrier Permeability and Neuronal Injury
}

\section{Michael M Gezalian}

Cedars-Sinai Medical Center https://orcid.org/0000-0003-3466-2814

\section{Padmesh Rajput}

Cedars-Sinai Medical Center

Nicklaus Sparrow

Cedars-Sinai Medical Center

Luigi Mangiacotti

Cedars-Sinai Medical Center

\section{Yosef Koronyo}

Cedars-Sinai Medical Center

Maya Koronyo-Hamaoui

Cedars-Sinai Medical Center

Shouri Lahiri ( $\square$ shouri.lahiri@csmc.edu )

Cedars-Sinai Medical Center https://orcid.org/0000-0002-5469-6467

\section{Research}

Keywords: Mechanical ventilation, cognitive impairment, Alzheimer's disease, critical illness, delirium, amyloid beta, encephalopathy, blood brain barrier breakdown

Posted Date: August 5th, 2020

DOI: https://doi.org/10.21203/rs.3.rs-52225/v1

License: (9) This work is licensed under a Creative Commons Attribution 4.0 International License. Read Full License 


\section{Abstract}

Background: Prior published data show that short-term mechanical ventilation contributes to pathological hallmarks of Alzheimer's Disease (AD) by increasing cerebral accumulation of the amyloid- $\beta_{1-40}\left(A \beta_{1-40}\right)$ peptide and neuroinflammation while unexpectedly decreasing blood-brain barrier permeability. In this study, we hypothesized that the acute cerebral immunochallenge of mechanical ventilation promotes physiological emergence of $A \beta_{1-40}$ to regions of acute vascular disruption, resulting in reduced neuronal injury.

Methods: Immunohistology was used to assess the regional relationship between cerebral $A \beta_{1-40}$ accumulation, acute vascular disruption, and neuronal inflammation and apoptosis as measured by cleaved caspase-3 (CC3) expression from mechanically ventilated double transgenic AD-model (ADtg) mice and compared to mechanically ventilated wild-type (WT) controls.

Results: There was significantly decreased expression of CC3 in regions of cortical and hippocampal A $\beta_{1}$. 40 accumulation in mechanically ventilated ADtg animals $(\mathrm{p}=0.0224)$. In contrast, $C C 3$ expression was significantly increased in cortical and hippocampal regions of mechanically ventilated WT controls $(p=0.0260) . A \beta_{1-40}$ accumulation occurred in regions of acute vascular disruption in a characteristic banding pattern and reduced blood-brain barrier permeability. The banding pattern was significantly increased in ADtg mice subjected to mechanical ventilation compared to non-mechanically ventilated ADtg mice $(p=0.0002)$. Additionally, no evidence of irreversible neurological injury is seen in mechanically ventilated ADtg and WT mice, as demonstrated by the absence of TUNEL positive staining.

Conclusions: This study provides first evidence that acute short-term mechanical ventilation-induced cerebral immunochallenge promotes physiological emergence of $A \beta_{1-40}$ to reduce blood-brain barrier permeability and neuronal injury. While it is expected that sustained or long-term activation of this normally protective pathway leads to dysregulated $A \beta_{1-40}$ accumulation, neurodegeneration, and cognitive impairment, these data suggest that a window of opportunity may exist within which implementation of timely interventions may limit cognition-relevant cerebral injury.

\section{Background}

Recent pre-clinical studies have shown that short-term mechanical ventilation contributes to pathophysiological hallmarks of Alzheimer's Disease (AD $)^{1-5}$. While these data provide mechanistic insights to explain the high rates of cognitive decline after mechanical ventilation, they reveal additional unexpected results. Despite an increase in both soluble $A \beta_{1-40}$ accumulation and cognition-relevant neuroinflammation, mechanical ventilation of double transgenic $A D(A D t g)$ mice significantly decreased cortical BBB permeability, while the same mechanical ventilation intervention significantly increased cortical BBB permeability in wild-type (WT) mice ${ }^{1,6}$. These findings were contrary to the a priori hypothesis, as BBB permeability was expected to be more significantly increased in mechanically ventilated ADtg mice who are considered more susceptible to acute systemic and cerebral stressors. 
Although the role of $A \beta$ has historically been considered largely pathological, more recent evidence suggests that the peptide may initially emerge to serve a protective function. One hypothesis proposes that $A \beta$ emerges as an innate immune response to cerebral immunochallenge and plays a key role to neutralize neuroinflammatory processes, entrap infectious pathogens, and prevent their entry across the BBB into neurons - thereby decreasing BBB permeability and preventing neurodegeneration ${ }^{7-11}$. While sustained or long-term activation of this normally protective pathway is thought to lead to dysregulated $A \beta$ accumulation, neurodegeneration, and cognitive decline, it is possible that during the acute phase of mechanical ventilation-induced cerebral immunochallenge, $A \beta$ serves a physiological and protective role to reduce BBB permeability and neuronal injury.

Accordingly, in this study, we sought to examine the hypothesis that 4 hours of mechanical ventilation of ADtg mice reduces neuronal injury and that the accumulation of $A \beta_{1-40}$ occurs in regions of acute vascular disruption and reduced neuronal injury.

\section{Methods}

\section{Mice}

ADtg mouse models from the B6Cg-Tg (APP SWE $\left._{\text {PSEN1 }}{ }_{\triangle E 9}\right)$ 85Dbo/J strains, and their sex- and agematched non-transgenic WT littermates, were purchased from Jackson Laboratories (MMRC stock \#34832-JAX|APP/PS1), then bred and maintained at Cedars-Sinai Medical Center in common

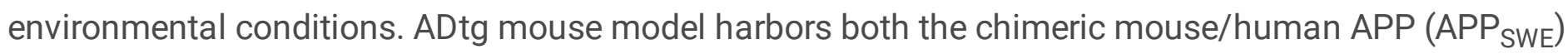
and the mutant human presenilin $1\left(P S E N 1_{\triangle E 9}\right)$ genes $[13,14]$. A total of 41 mice (4 to 6.5 months old and 24.1 to $29.7 \mathrm{~g}$ in weight) were used: 21 ADtg mice and 20 WT mice. Of the ADtg mice, 12 were mechanically ventilated and 9 were not mechanically ventilated (spontaneous breathing) and of the 20 WT mice, 8 were mechanically ventilated and 12 were not mechanically ventilated. All experiments were conducted in accordance with Cedars-Sinai Medical Center Institutional Animal Care and Use Committee (IACUC) guidelines under an approved protocol and complied with the current US law. Final analysis and data are representative of remaining samples from mice after exclusion of certain samples due to tissue availability and integrity.

\section{Genotyping}

Genomic DNA was extracted from a tail or ear tip using a DNA extraction kit (Qiagen) following the manufacturer's protocol. Mice used in this study were genotyped for the presence of the transgenes by polymerase chain reaction, as previously described.

\section{Intubation and mechanical ventilation}

Mice to be mechanically ventilated were anesthetized via intraperitoneal injection of a mix of ketamine (Vedco Inc., Saint Joseph, MO, USA) and dexmedetomidine (Pfizer, Irvine, CA, USA) (75 mg/kg and $0.5 \mathrm{mg} / \mathrm{kg}$, respectively). Mice were orotracheally intubated and ventilated for 4 hours using an Inspira 
volume-controlled small animal ventilator (Harvard Apparatus, Holliston, MA, USA) with a tidal volume of $15 \mathrm{cc} / \mathrm{kg}$ and a respiratory rate of 70 breaths per minute, with zero positive end-expiratory pressure. Subcutaneous phosphate buffered saline (PBS) $(0.5 \mathrm{cc})$ was administered at the onset of mechanical ventilation and every 3 hours of mechanical ventilation thereafter. Fifty milligrams per kilogram of ketamine was administered via subcutaneous injections as needed to maintain sedation, usually every 2-3 hours. Mice were kept warm on a $38^{\circ} \mathrm{C}$ heating pad (Hallowell EMC, Pittsfield, MA, USA). Anesthesia was reversed with atipamezole ( $1 \mathrm{mg} / \mathrm{kg}$ in $100 \mu \mathrm{L}$ of sterile water), and the animals were allowed to recover in their cages on a heating pad for 6 hours. Control mice remained in their cages until the end of mechanical ventilation, at which time they were euthanized together with the ventilated mice.

\section{Tracer infusion to assess BBB permeability}

Following mechanical ventilation, mice recovered for 6 hours after which each was administered $50 \mu \mathrm{L}$ of intravenous fluorescein isothiocyanate (FITC) -dextran $2000 \mathrm{kD}(0.25 \%)$ via tail vein and subsequently euthanized by perfusion 30 minutes later. Non-mechanically ventilated control animals also underwent tracer infusion and euthanasia by perfusion 30 minutes later.

\section{Immunohistochemistry and Microscopy}

At experimental completion, mice were deeply anesthetized and perfused with ice-cold saline supplemented with $0.5 \mathrm{mM}$ Ethylenediaminetetraacetic acid. Right brain hemispheres were isolated and fixed in $2.5 \%$ paraformaldehyde (Sigma-Aldrich), then cryo-protected in $30 \%$ sucrose prior to embedding in optimal cutting compound. Free floating, 30- $\mu \mathrm{m}$-thick coronal brain cryosections were prepared and stored at $4{ }^{\circ} \mathrm{C}$ in PBS $+0.02 \%$ sodium azide until staining. Brain sections were affixed to slides by air drying, and then subjected to heat induced epitope retrieval for 5 minutes in antigen-retrieval solution $(\mathrm{pH}$ 6.0, Dako) prior to permeabilization/blocking in $5 \%$ bovine serum albumin $+0.3 \%$ Triton X-100 in PBS ( 1 hour at RT). Sections were incubated overnight at $4{ }^{\circ} \mathrm{C}$ with rabbit anti-cleaved caspase-3 (CC3) pAb (CC3; 1:800; Cell Signaling). After washing, sections were incubated with a combination of AF + 555-conjugated donkey anti-rabbit secondary antibody (1:500; Thermo Fisher Scientific), and AF647-labeled mouse antihuman $A \beta_{1-40} \mathrm{mAb}$ [clone 11A50-B10; specific for $A \beta$ isoform ending at the 40th amino acid (1:50; Covance)] ( $2 \mathrm{~h}$ at RT). The anti-A $\beta_{1-40} \mathrm{mAb}$ was pre-labeled using the Zenon AF647 Mouse lgG1 Labeling Kit (Thermo Fisher Scientific) according to the manufacture's protocol at 6:1 molar ratio of AF647conjugated Fab to anti-A $\beta_{1-40}$ antibody. After washing, sections were incubated in $0.1 \%$ sudan black in $70 \%$ ethanol for $30 \mathrm{~min}$ to reduce tissue autofluorescence. Sections were mounted using ProLong Glass with 4',6-diamidino-2-phenylindole (DAPI) (Thermo Fisher Scientific). Negative controls were processed using the same protocol with the omission of the primary antibody to assess non-specific labeling. A Carl Zeiss Axiolmager Z.2 fluorescence microscope equipped with an Apotome 2.0 and an Axiocam HRm camera, and controlled by Zen Blue Pro (version 2.3) software was used to acquire and process images. Images for CC3 analysis were acquired with a 10x objective (NA 0.3, Zeiss) as a $5 \times 5$ tiled image that encompassed both the neocortex and hippocampus of each section. Images for $A \beta_{1-40}$ banding analysis were acquired with a $20 x$ objective (NA 0.8 , Zeiss) as single-plane fields, but representative images in Fig. 2 are maximum intensity projections of a $8 \mu \mathrm{m}$ z-stack. 


\section{Image and statistical analysis}

Fiji and Prism 8.3.0 (GraphPad) software was used for image and statistical analysis, respectively. Three coronal sections containing the hippocampus and adjacent cortex per animal were analyzed (ventral, mid, and dorsal). For CC3 analysis, a $400 \mu \mathrm{m}^{2}$ region of interest (ROI) was drawn around the frontal neocortex (beginning at the midline), and the number of positive pixels was measured and expressed as percent area of the ROI. Values from the three sections were averaged to yield one number per animal. For $A \beta_{1-40}$ band analysis, three images per section including FITC positive blood vessels along the cortex were analyzed. The number of bands seen per image ( 9 total) were summed to yield one number per animal. Un-paired $T$ test between spontaneously breathing and mechanically ventilated mice within each strain was used to determine statistical significance. Welch's T-test was used where standard deviations were determined to be unequal.

\section{Results}

A brief summary of our previously published data is as follows: there was a significant increase in inflammatory cytokine concentrations such as TNF-a and IL-6 in all mechanically ventilated animals, both WT and ADtg $(n=21)$. Additionally, there was increased BBB permeability, measured by increased accumulation of high molecular FITC-dextran, in mechanically ventilated WT mice brain tissue $(n=12)$. In contrast, despite increased accumulation of cerebral soluble $A \beta_{1-40}$, there was a significant $(p=0.02)$ decrease in BBB permeability, observed by decreased accumulation of FITC-dextran in mechanically ventilated ADtg mice $(n=9)$.

To determine how the accumulation of soluble $A \beta_{1-40}$ and decrease in BBB permeability may affect neurons, we quantified the relationship between $A \beta_{1-40}$ accumulation and a marker of neuronal apoptosis and inflammation, CC3. During apoptosis, various signaling pathways converge on the activation (cleavage) of caspase-3, which in turn activates other apoptotic pathways that lead to genomic breakdown, ultimately precipitating cell death. CC3 expression in frontal and parietal cortices, particularly in layers 1,2 , and 4 , was significantly increased in WT mice that underwent mechanical ventilation with $15 \mathrm{cc} / \mathrm{kg}$ tidal volumes when compared to mice that underwent spontaneous breathing $(p=0.0260)$ (Fig. 1, Section A).

Analysis of CC3 accumulation in frontal and parietal cortices of ADtg mice that underwent spontaneous breathing and mechanical ventilation demonstrated significantly decreased CC3 in the frontal and parietal cortices of ADtg mice that underwent mechanical ventilation compared to spontaneous breathing animals $(p=0.0224)$ (Fig. 1, Section $B)$. To assess for irreversible neuronal injury by detecting DNA fragmentation within cortical and hippocampal neurons, terminal deoxynucleotidyl transferase dUTP nick end labeling (TUNEL) staining was utilized (Fig. 1, Section C). There was no evidence of TUNEL staining in both mechanically ventilated and spontaneously breathing ADtg or WT mice.

FIGURE 1. CC3 expression in the cortices of WT and ADtg mice following mechanical ventilation (MV). 
a. WT mice: Increased CC3 expression in MV WT mice. Images of coronal brain sections presented here demonstrating the expression of CC3 (green) and nucleus stain DAPI (blue). Magnified images of cortex (representative location highlighted via dotted box) included. CC3 was measured in frontal cortex of WT mice subjected to MV and compared to spontaneously breathing (SB) group. Increased expression of CC3 is observed in layers 1,2, and 4 of cortex in mice subjected to MV. A significant increase of CC3 expression in MV group is seen when compared to SB group $(p=0.0260)$.

\section{b. ADtg mice: Decreased expression of $C C 3$ and accumulation of $A \beta_{1-40}$ in the frontal and parietal} cortices in the ADtg mice. Images of coronal brain sections presented here demonstrating the expression of CC3 (green) and nucleus stain DAPI (blue). Magnified images of cortex (representative location highlighted via dotted box) included. ADtg mice were subjected to MV and compared to $S B$ ADtg controls. A decreased expression of CC3 in the cortex is seen in MV group when compared to SB control $(p=0.0224)$.

c. TUNEL stained sections of MV and SB WT and ADtg mice: Sections demonstrate absence of the TUNEL staining (a control section included for comparison in a form of TUNEL staining in a mouse with a stroke).

Further microscopic analysis revealed accumulation of soluble $A \beta_{1-40}$ along the vascular membrane in a characteristic banding pattern within regions of acute vascular disruption. There was a significant increase in banding pattern in mechanically ventilated ADtg mice compared to non-mechanically ventilated $A D$ tg controls $(p=0.0002)$ (Fig. 2). Further, regions of $A \beta_{1-40}$ accumulation in mechanically ventilated $A D t g$ mice appeared to have less $B B B$ permeability, whereas regions with devoid of $A \beta_{1-40}$ demonstrated elevated FITC-dextran extravasation, implying increased vascular permeability.

FIGURE 2. $A \boldsymbol{\beta}_{1-40}$ and FITC-dextran stained sections demonstrating banding patters of $A \boldsymbol{\beta}_{1-40}$ in areas of vascular disruption in MV ADtg mice. Panel $A$ shows a lack of $A \beta_{1-40}$ within a region of FITC-dextran accumulation in SB ADtg mice. Panel $B$, shows a MV ADtg mouse brain parenchyma with markedly increased distribution of $A \beta_{1-40}$, in a "banding" pattern within a region of acute vascular disruption. The $A \beta_{1-40}$ interrupts the FITC-dextran within the blood vessel (arrows indicate $A \beta_{1-40}$ staining). Panel $C$, taken from the same mouse subjected to MV, demonstrates extravasation of FITC-dextran from within the blood vessel to the brain parenchyma in a region without $A \beta_{1-40}$ staining. $A$ significant increase in $A \beta_{1-40}$ bands/\% area of FITC in ADtg mice subjected to MV compared to SB ADtg mice $(p=0.0002)$.

\section{Discussion}

This study provides first evidence of a physiological and protective role of $A \beta_{1-40}$ during the acute cerebral stress of mechanical ventilation as evidenced by both reduced initiation of neuronal apoptosis and increased distribution of $A \beta_{1-40}$ to regions of acute vascular disruption in frontal and parietal cortices. These findings are overall consistent with the Antimicrobial Protection Hypothesis of AD, which proposes that $A \beta$ initially emerges as an innate immune response to protect from immune-mediated injury to the brain. 
Cerebral accumulation of the $A \beta$ peptide is widely considered a pathological hallmark of $A D^{12}$. Although $A \beta$ deposition has been shown to induce neuroinflammation and cognitive impairment, the process by which cerebral $A \beta$ accumulates in the brain remains unclear ${ }^{10}$. Further, recent studies demonstrate a bidirectional relationship between neuroinflammation and $A \beta$ deposition- an increased $A \beta$ expression and accumulation occurs as a normal physiological and protective response to perceived or actual threat of cerebral immune-related injury ${ }^{10}$. Specifically, these studies show that: 1 ) disruption of immune pathways attenuates $A \beta$ deposition ${ }^{13}$, 2) synthetic $A \beta$ inhibits growth of common pathogens, such as gram-positive and gram-negative bacteria, Candida albicans, influenza A virus, and herpes simplex virus type 1 , by up to 200 -fold in vitro $7,8,10,11,14,3$ ) expression of human $A \beta$ protects and increases survival in transformed cell culture and transgenic $C$. elegans and mouse infection models ${ }^{9}$, and 4) antimicrobial activity in AD whole brain homogenates are higher compared to aged matched non-AD samples; this antimicrobial activity correlates with tissue $A \beta$ levels ${ }^{11}$. The findings of our research extend the scope of these studies to include short-term mechanical ventilation-induced cerebral immunochallenge as a possible precipitant of $A \beta_{1-40}$ accumulation which, when sustained or activated over a long-term period, may lead to dysregulated and excessive cortical $A \beta$ accumulation and deposition triggering neuroinflammatory cascades leading to neurodegeneration and cognitive impairment ${ }^{10}$.

Our study results further suggest the presence of a window of opportunity to prevent cognition-relevant neuronal injury in those with pre-existing AD pathology. Neuronal injury via signal cascades leading to neuronal apoptosis and inflammation, is initiated by increased activation of pro caspase- 3 via cleavage to create CC3. The decreased accumulation of CC3 in the cortices of mechanically ventilated ADtg mice with $A \beta_{1-40}$ suggests a protective effect of $A \beta_{1-40}$ on neuronal injury. However, the lack of irreversible neuronal injury, as evidenced by the absence of TUNEL positive staining in the frontal and parietal cortices of WT animals, is consistent with the clinical presentation of delirium, which is thought to be a harbinger of long-term cognitive decline, after mechanical ventilation. Delirium is a potentially reversible acute confusional state that is thought to reflect frontal and parietal cortical dysfunction and is strongly associated with mechanical ventilation ${ }^{2,15-17}$. These data provide evidence showing that short-term mechanical ventilation may directly injure cortical structures that are presumed to underlie delirium and suggest that a timely reversal of this neuropathology may improve long-term cognitive outcomes after critical illness.

This study has important limitations to consider. The use of a high dose mechanical ventilation, i.e. $15 \mathrm{cc} / \mathrm{kg}$ tidal volume, may not reflect current clinical practice as lower tidal volume ventilation is generally considered the standard of care. However, the duration of mechanical ventilation was only 4 hours and the animals did not have any concurrent systemic infections that could compound to the cerebral injury. The finding that mechanical ventilation independently contributes to cognition-relevant neuropathological changes is expected to have translational relevance. Further, while it may seem counterintuitive that the presence of pre-existing AD pathology confers neuroprotection, the ADtg mice were only 5 months old and pre-symptomatic, and accordingly do not reflect the cognitively impaired aged patient cohort who are at highest risk for cognitive decline after critical illness. Future studies are 
needed to assess cerebral effects of mechanical ventilation in older and symptomatic ADtg mice with cognitive impairment.

\section{Conclusion}

In conclusion, this study provides first evidence that acute short-term mechanical ventilation-induced cerebral immunochallenge promotes physiological emergence of $A \beta_{1-40}$ to reduce BBB permeability and neuronal injury. While it is expected that sustained or long-term activation of this normally protective pathway leads to dysregulated $A \beta_{1-40}$ accumulation, neurodegeneration, and cognitive impairment, these data suggest that a window of opportunity may exist within which implementation of timely interventions may limit cognition-relevant cerebral injury. This study also provides additional evidence that mechanical ventilation injures frontal and parietal cortices structures that are implicated in the pathogenesis of delirium. This is consistent with the preponderance of clinical data that associate mechanical ventilation with delirium.

\section{Declarations}

Ethics approval and consent to participate: All experiments were conducted in accordance with CedarsSinai Medical Center Institutional Animal Care and Use Committee (IACUC) guidelines under an approved protocol and complied with current United States law.

Consent for publication: Not applicable

Availability of data and materials: The data that support the findings of this study are available from the corresponding authors upon request.

Competing interests: The authors declare that they have no competing interests.

Funding: NIH/NIA R03AG064106, American Academy of Neurology Institute (SL)

Authors' contributions: SL contributed to the study concept and design. SL, NS, MG, LM, YK, PSR, and MKH contributed to the acquisition of data. SL, NS, MG and PSH did the analysis and interpretation of data. MG and SL drafted the manuscript. NS did the statistical analysis. SL did the study supervision. All authors read and approved the final manuscript.

Acknowledgements: None

Authors' Information: ${ }^{1}$ Department of Neurology, Cedars-Sinai Medical Center, 127 S. San Vicente Blvd., AHSP Building, Suite A6600, A8103, Los Angeles, CA 90048, USA. ²Department of Neurosurgery, CedarsSinai Medical Center, 127 S. San Vicente Blvd., AHSP Building, Suite A6600, A8103, Los Angeles, CA 90048, USA. ${ }^{3}$ Department of Biomedical Sciences, Cedars-Sinai Medical Center, 127 S. San Vicente Blvd., AHSP Building, Suite A6600, A8103, Los Angeles, CA 90048, USA. 


\section{Abbreviations}

Alzheimer's Disease - AD

Amyloid beta- $A \beta$

Blood-brain barrier - BBB

Chimeric mouse/human APP - APP SWE

Cleaved caspase-3 - CC3

4',6-diamidino-2-phenylindole - DAPI

Double transgenic AD - ADtg

Fluorescein isothiocyanate - FITC

Mechanical ventilation - MV

Mutant human presenilin 1 - PSEN1 ${ }_{\Delta E} 9$

Region of interest - ROI

Spontaneous breathing - SB

Terminal deoxynucleotidyl transferase dUTP nick end labeling - TUNEL

Wild-type - WT

\section{References}

1. Lahiri S, Regis GC, Koronyo Y, et al. Acute neuropathological consequences of short-term mechanical ventilation in wild-type and Alzheimer's disease mice. Crit Care. 2019;23:63.

2. Sasannejad C, Ely EW, Lahiri S. Long-term cognitive impairment after acute respiratory distress syndrome: a review of clinical impact and pathophysiological mechanisms. Crit Care. 2019;23:352.

3. Chen C, Zhang Z, Chen T, Peng M, Xu X, Wang Y. Prolonged mechanical ventilation-induced neuroinflammation affects postoperative memory dysfunction in surgical mice. Crit Care. 2015;19:159.

4. Gonzalez-Lopez A, Lopez-Alonso I, Aguirre A, et al. Mechanical ventilation triggers hippocampal apoptosis by vagal and dopaminergic pathways. Am J Respir Crit Care Med. 2013;188:693-702.

5. Bilotta F, Giordano G, Sergi PG, Pugliese F. Harmful effects of mechanical ventilation on neurocognitive functions. Crit Care. 2019;23:273. 
6. Giordano G, Pugliese F, Bilotta F. Mechanical ventilation and long-term neurocognitive impairment after acute respiratory distress syndrome. Crit Care. 2020;24:30.

7. Bourgade K, Garneau H, Giroux G, et al. beta-Amyloid peptides display protective activity against the human Alzheimer's disease-associated herpes simplex virus-1. Biogerontology. 2015;16:85-98.

8. Bourgade K, Le Page A, Bocti C, et al. Protective Effect of Amyloid-beta Peptides Against Herpes Simplex Virus-1 Infection in a Neuronal Cell Culture Model. J Alzheimers Dis. 2016;50:1227-41.

9. Kumar DK, Choi SH, Washicosky KJ, et al. Amyloid-beta peptide protects against microbial infection in mouse and worm models of Alzheimer's disease. Sci Transl Med. 2016;8:340ra72.

10. Moir RD, Lathe R, Tanzi RE. The antimicrobial protection hypothesis of Alzheimer's disease. Alzheimer's dementia: the journal of the Alzheimer's Association. 2018;14:1602-14.

11. Soscia SJ, Kirby JE, Washicosky KJ, et al. The Alzheimer's disease-associated amyloid beta-protein is an antimicrobial peptide. PLoS One. 2010;5:e9505.

12. Tarasoff-Conway JM, Carare RO, Osorio RS, et al. Clearance systems in the brain-implications for Alzheimer diseaser. Nat Rev Neurol. 2016;12:248.

13. Reed-Geaghan EG, Reed QW, Cramer PE, Landreth GE. Deletion of CD14 attenuates Alzheimer's disease pathology by influencing the brain's inflammatory milieu. J Neurosci. 2010;30:15369-73.

14. White MR, Kandel R, Tripathi S, et al. Alzheimer's associated beta-amyloid protein inhibits influenza A virus and modulates viral interactions with phagocytes. PLoS One. 2014;9:e101364.

15. Bulic D, Bennett $M$, Rodgers $\mathrm{H}$, et al. Delirium After Mechanical Ventilation in Intensive Care Units: The Cognitive and Psychosocial Assessment (CAPA) Study Protocol. JMIR research protocols. 2017;6:e31.

16. Girard TD, Jackson JC, Pandharipande PP, et al. Delirium as a predictor of long-term cognitive impairment in survivors of critical illness. Crit Care Med. 2010;38:1513-20.

17. Turon M, Fernandez-Gonzalo S, de Haro C, Magrans R, Lopez-Aguilar J, Blanch L. Mechanisms involved in brain dysfunction in mechanically ventilated critically ill patients: implications and therapeutics. Annals of translational medicine. 2018;6:30.

\section{Figures}


A

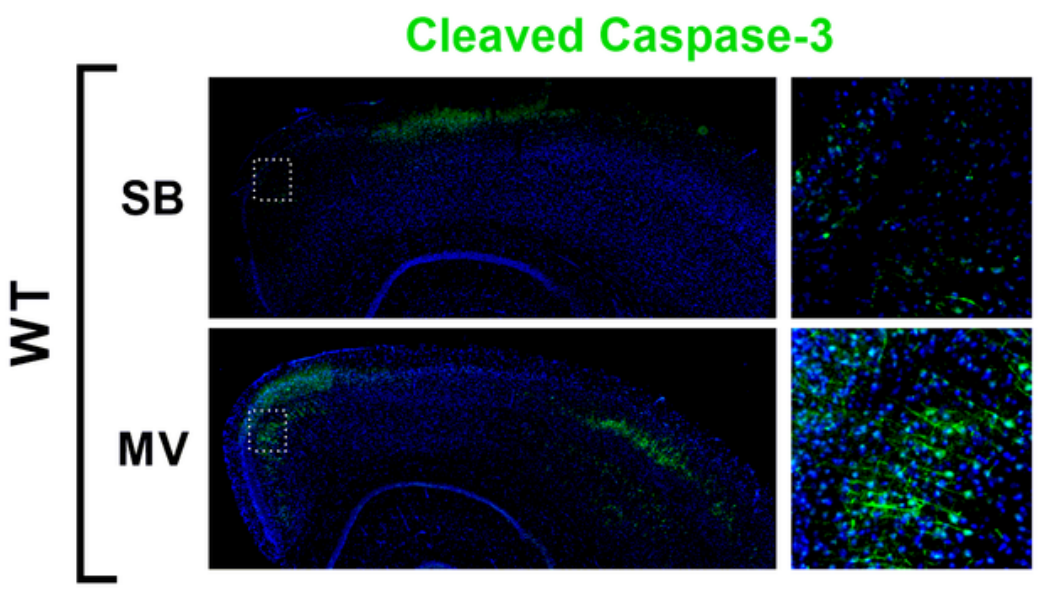

B

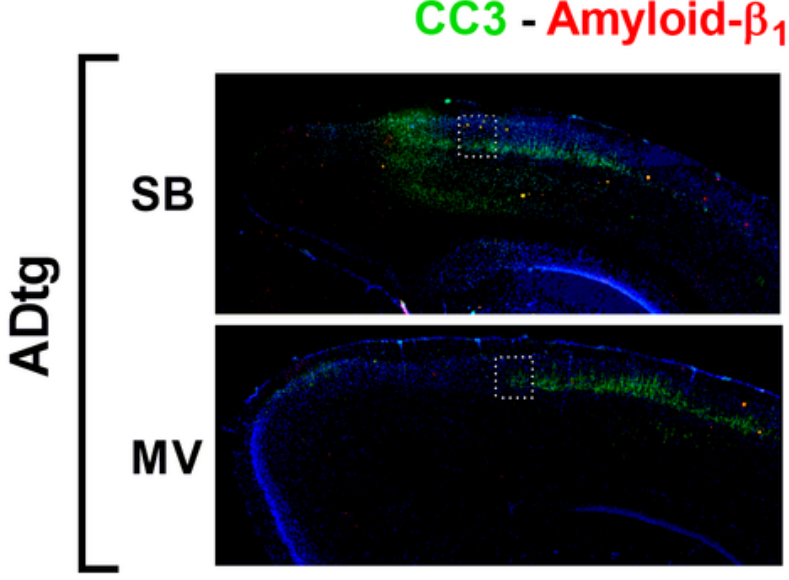

C
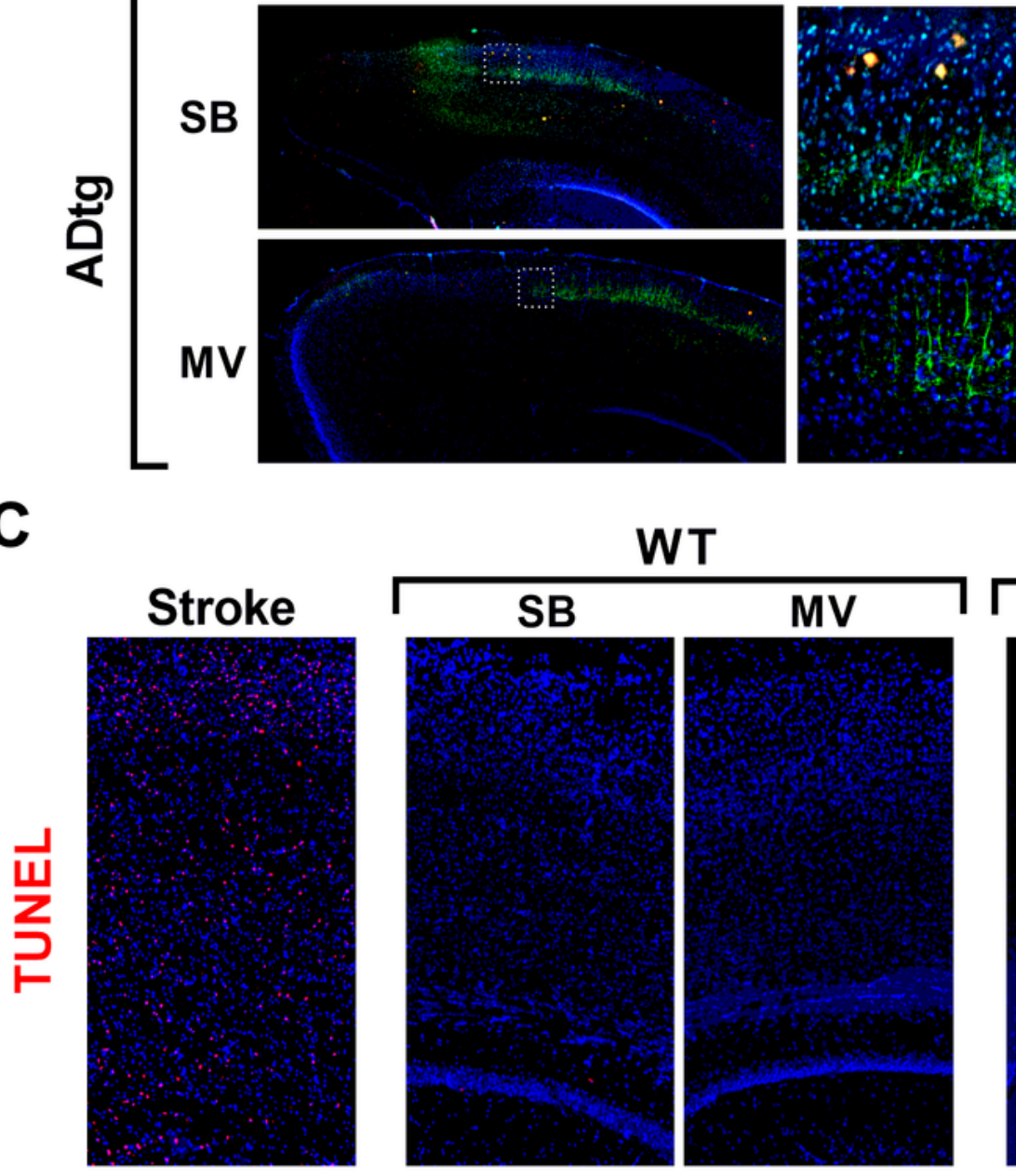

WT

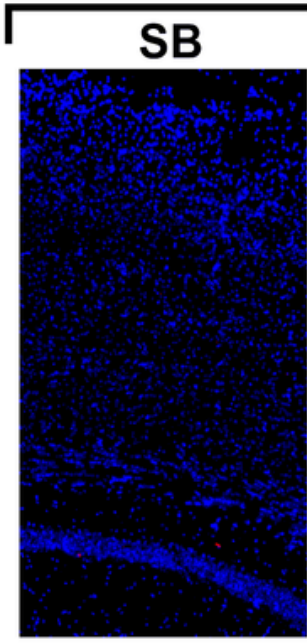

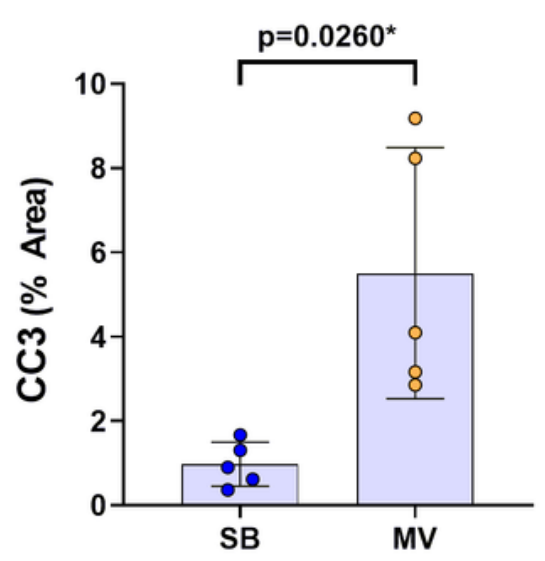

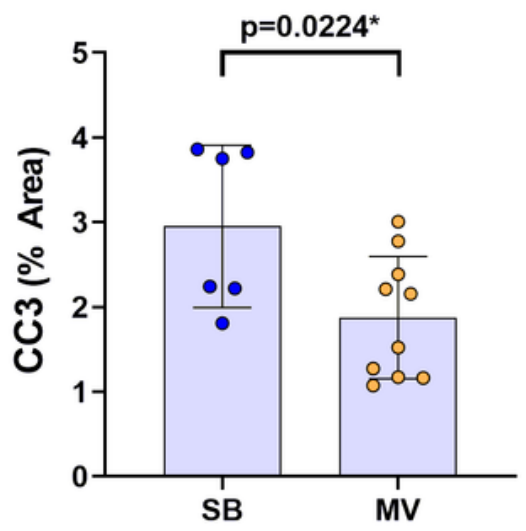

ADtg

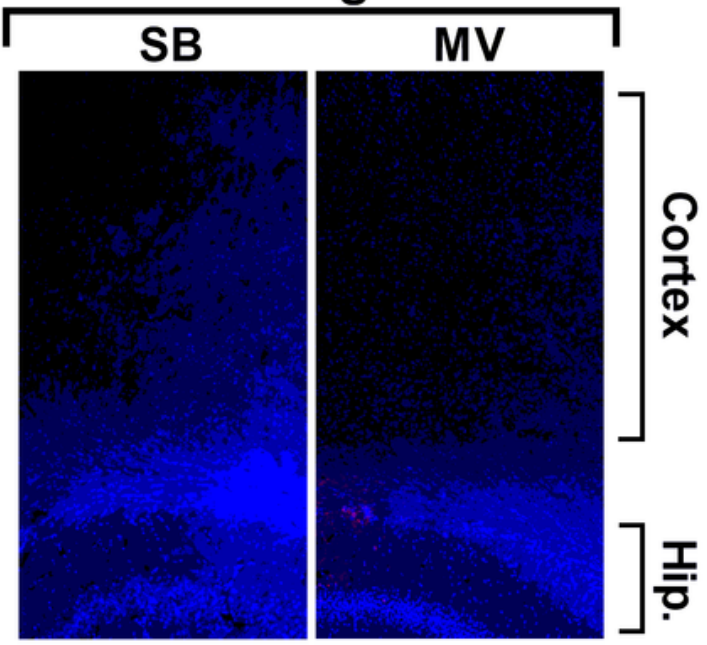

Figure 1

CC3 expression in the cortices of WT and ADtg mice following mechanical ventilation (MV). A. WT mice: Increased CC3 expression in MV WT mice. Images of coronal brain sections presented here demonstrating the expression of CC3 (green) and nucleus stain DAPI (blue). Magnified images of cortex (representative location highlighted via dotted box) included. CC3 was measured in frontal cortex of WT mice subjected to MV and compared to spontaneously breathing (SB) group. Increased expression of 
CC3 is observed in layers 1, 2, and 4 of cortex in mice subjected to MV. A significant increase of CC3 expression in MV group is seen when compared to SB group ( $p=0.0260)$. B. ADtg mice: Decreased expression of CC3 and accumulation of AB1-40 in the frontal and parietal cortices in the ADtg mice. Images of coronal brain sections presented here demonstrating the expression of CC3 (green) and nucleus stain DAPI (blue). Magnified images of cortex (representative location highlighted via dotted box) included. ADtg mice were subjected to MV and compared to SB ADtg controls. A decreased expression of CC3 in the cortex is seen in MV group when compared to SB control $(p=0.0224)$. C. TUNEL stained sections of MV and SB WT and ADtg mice: Sections demonstrate absence of the TUNEL staining (a control section included for comparison in a form of TUNEL staining in a mouse with a stroke).
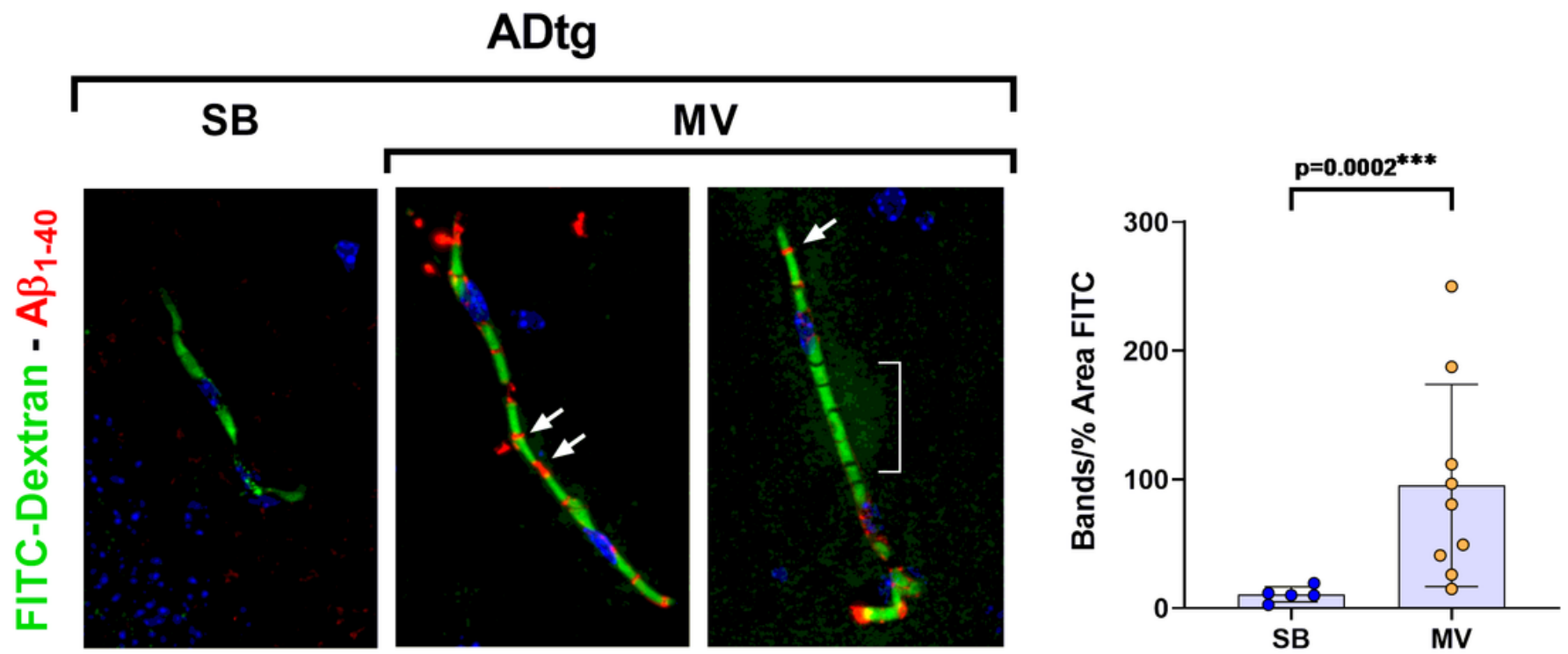

Figure 2

A $1-40$ and FITC-dextran stained sections demonstrating banding patters of $A \beta 1-40$ in areas of vascular disruption in MV ADtg mice. Panel A shows a lack of AB1-40 within a region of FITC-dextran accumulation in SB ADtg mice. Panel B, shows a MV ADtg mouse brain parenchyma with markedly increased distribution of $A \beta 1-40$, in a "banding" pattern within a region of acute vascular disruption. The A 1-40 interrupts the FITC-dextran within the blood vessel (arrows indicate A 1-40 staining). Panel C, taken from the same mouse subjected to MV, demonstrates extravasation of FITC-dextran from within the blood vessel to the brain parenchyma in a region without $A \beta 1-40$ staining. $A$ significant increase in $A \beta 1-$ 40 bands $/ \%$ area of FITC in ADtg mice subjected to MV compared to SB ADtg mice $(p=0.0002)$. 